\title{
Assessment of stress and its risk factors among primary school teachers in the Klang Valley, Malaysia
}

\begin{abstract}
Introduction: This cross-sectional study determined the workplace stressors, stress levels, mental health status and their influencing factors, among primary school teachers in the Klang Valley, Malaysia. Methodology: Nine primary schools in Klang Valley which fulfil the inclusive criteria were randomly selected from a list obtained from the Ministry of Education website. Two hundred and seventy two teachers from the selected school, volunteered to participate in the study. A questionnaire was used to determine socio-demographic background, working information and medical history. Teacher Stress Inventory was used to measure the stressor and stress levels; while General Health Questionnaire was used to measure the mental health status. Result: Results showed that most of the teachers experience moderate stress level $(71.7 \%)$ and only $12.1 \%$ had low mental health status. Student misbehaviour was the main stressor in the school environment (mean=2.62). Gender $(\mathrm{p}=0.001)$ and workload $(\mathrm{p}=0.002)$ showed a significant contributing factors toward mental health status. Conclusion: These primary school teachers experience stress mainly due to the student misbehaviour and the mental well-being were influenced by the workload and gender. Women teachers with heavy workload had relationship with lower mental health status.
\end{abstract}

Keyword: Primary school teachers; Stress; Teacher stress inventory; Mental health status 